\title{
Modelling slight compressibility for hyperelastic anisotropic materials
}

\author{
J.G. Murphy*ab, G.A. Rogerson ${ }^{c}$ \\ ${ }^{a}$ Department of Mechanical Engineering, \\ Dublin City University, Glasnevin, Dublin D09 W6Y4, Ireland. \\ ${ }^{b}$ School of Mathematics, Statistics, and Applied Mathematics, \\ National University of Ireland Galway, \\ University Road, Galway, Ireland.
}

${ }^{c}$ School of Computing and Mathematics, Keele University, Keele, Staffordshire, ST5 5BG, U.K.

* corresponding author. email: jeremiah.murphy@dcu.ie, phone: +353-1-700-8924

\begin{abstract}
In order to avoid the numerical difficulties in locally enforcing the incompressibility constraint using the displacement formulation of the Finite Element Method, slight compressibility is typically assumed when simulating the mechanical response of nonlinearly elastic materials. The current standard method of accounting for slight compressibility of hyperelastic materials assumes an additive decomposition of the strain-energy function into a volumetric and an isochoric part. A new proof is given to show that this is equivalent to assuming that the hydrostatic stress is a function of the the volume change only and that uniform dilatation is a possible solution to the hydrostatic stress boundary value problem, with therefore no anisotropic contribution to the mechanical response. An alternative formulation of slight compressibility is proposed, one that does not suffer from this defect. This new model generalises the standard model by including a mixed term in the volume change and isochoric response. Specific models of slight compressibility are given for isotropic, transversely isotropic and orthotropic materials.
\end{abstract}

Keywords: nonlinearly elastic materials; slightly compressible; volumetric-isochoric split. 


\section{Introduction}

The Finite Element Method (FEM) is the preeminent numerical method when simulating the mechanical response of non-linearly elastic materials. The use of the FEM when modelling soft tissue has focussed attention on the accounting for the slight compressibility of such tissue, especially as to how it is incorporated in commercial and open-source Finite Element codes, which are used in the vast majority of simulations. Without exception, slight compressibility is modelled in these codes by assuming that the strain energy function per unit undeformed volume $W$ can be additively decomposed into separate volumetric and isochoric components as follows:

$$
W\left(J, \boldsymbol{C}^{*}\right)=f(J)+\mathcal{W}\left(\boldsymbol{C}^{*}\right)
$$

where $J$ is the determinant of the deformation gradient tensor $\boldsymbol{F}$ and $\boldsymbol{C}^{*}$ is the isochoric right Cauchy-Green strain tensor defined by $\boldsymbol{C}^{*}=J^{-2 / 3} \boldsymbol{C}, \boldsymbol{C} \equiv \boldsymbol{F}^{T} \boldsymbol{F}$, noting that $\operatorname{det} \boldsymbol{C}^{*}=1$ (see, for example, Flory [1]). Typically the incompressibility constraint is to be relaxed for a known incompressible strain energy $W_{i}(\boldsymbol{C})$, with therefore

$$
W(1, \boldsymbol{C})=W_{i}(\boldsymbol{C})
$$

and so here $\mathcal{W}\left(\boldsymbol{C}^{*}\right)=W_{i}\left(\boldsymbol{C}^{*}\right)$, assuming that $f(1)=0$. Ideally $W_{i}(\boldsymbol{C})$ should be compatible with the corresponding linear theory, as it seems that a well-posed non-linear model should fully recover the linear on restriction to infinitesimal values of the independent variable. Although not necessary for what follows, it will be assumed here that this compatibility is satisfied.

The assumption (1.1) is typically given without motivation; the primary reasons for its widespread use seem to be an intuitive appeal and mathematical convenience. Another reason for its widespread use when modelling anisotropic soft tissue could be its success in modelling isotropic elastomers, for which the decomposition (1.1) was originally proposed; 
it seems that the isotropic formulation of (1.1) was generalised in a natural way for anisotropic materials, without much consideration being paid as to how appropriate this is. This is the main focus here.

It is shown that the following are consequences of assuming (1.1) for all anisotropic materials:

- The corresponding linear theory cannot be fully recovered from the non-linear theory on restriction to infinitesimal strains (Federico [2]). It is axiomatic that any non-linear theory should recover the linear on restriction to infinitesimal values of the independent variable(s) (Quintanilla and Saccomandi [3]).

- Uniform dilatation is a solution to the boundary value problem of hydrostatic tension. This is the solution that is simulated in FEM codes (Ní Annaidh et al. [4]) and thus the fibres play no role in the mechanical response in this problem, which is surely physically unrealistic.

- The volumetric-isochoric split is equivalent to assuming that the trace of the Cauchy stress is a function only of the volume change (Charrier et al. [5]). Although the intuitive appeal of (1.1) is obvious, its equivalent formulation in terms of the stress lacks this appeal. Is it reasonable to postulate a theory of slight compressibility for anisotropic materials based on the assumption of isotropic response under hydrostatic tension?

Attention has already been drawn to these deficiencies for specific forms of anisotropy by, for example, Ní Annaidh et al. [4], Vergori et al. [6], Gilchrist et al. [7] and Nolan et al. [8]. The novelty here is that all results are obtained in complete generality, irrespective of material symmetry. Somewhat surprisingly, it is shown that proof of the above listed results for a general elastic material is trivial in comparison to the existing proofs for specific anisotropic models, such as those obtained by Sansour [9] and Horgan and Murphy [10] for example. 
The inability of models incorporating the volumetric-isochoric split (1.1) to capture physically realistic effects in hydrostatic tension and compression is the core element of the difficulties listed above. It might be argued that this inability is not important when simulating the mechanical response of slightly compressible materials in typical applications. However, when dealing with both complex geometries and boundary conditions, it is impossible to rule out at least local states of hydrostatic tension and compression and therefore accurate accounting for the behaviour of slightly compressible materials in these experiments is essential. An alternative approach to resolving these difficulties in compressible elasticity could be the simulation of perfect incompressibility instead. However, this is not an option, even if the numerical difficulties that this poses can be overcome in an efficient and accurate manner, as no real material is perfectly incompressible and slight compressibility is a fundamental aspect of the physical response of materials that have classically been modelled as being perfectly incompressible, such as soft tissue.

An alternative formulation of slight compressibility is suggested here in order to overcome the difficulties associated with (1.1). It seems sensible to generalise this formulation of slight compressibility in order to utilise the vast computational infrastructure already developed that is based on the volumetric-isochoric split. The approach proposed here is based on truncating a Taylor series in the volume change after the second order, as initially suggested by Spencer [11]. The zero order term is a perfectly incompressible material, assumed known from standard material characterisation tests. The first and second order coefficients need to be specified. Motivated by mathematical convenience, it will be assumed that the first order term is linear in the appropriate invariants of $\boldsymbol{C}^{*}$ and that the second order term is a positive constant. This has the intuitive appeal of a deceasing complexity in the $\boldsymbol{C}^{*}$ terms as the order of the Taylor series in $J$ increases. The current standard formulation of slight compressibility is a special case of this new approach, with (1.1) recovered if the linear term in the volume change is identically zero. Explicit models are proposed for modelling slightly compressible for isotropic, transversely isotropic and orthotropic materials. 
94

95 96 by

$$
\hat{W}(\boldsymbol{I})=W(1, \boldsymbol{I})=0,
$$

to ensure zero strain energy in the reference configuration, where here, and in what follows, $\boldsymbol{I}$ denotes the appropriate second-order identity tensor. Noting that

$$
\frac{\partial J}{\partial \boldsymbol{C}}=\frac{1}{2} J \boldsymbol{C}^{-1}, \quad \frac{\partial \boldsymbol{C}^{*}}{\partial \boldsymbol{C}}=-\frac{1}{3} J^{-2 / 3} \boldsymbol{C} \otimes \boldsymbol{C}^{-1}+J^{-2 / 3} \frac{\partial \boldsymbol{C}}{\partial \boldsymbol{C}},
$$

the constitutive law (2.1) can therefore be rewritten in the form

$$
\begin{aligned}
\boldsymbol{\sigma} & =\frac{2}{J} \boldsymbol{F}\left(\frac{\partial W}{\partial J} \frac{\partial J}{\partial \boldsymbol{C}}+\frac{\partial W}{\partial \boldsymbol{C}^{*}} \frac{\partial \boldsymbol{C}^{*}}{\partial \boldsymbol{C}}\right) \boldsymbol{F}^{T} \\
& =\frac{\partial W}{\partial J} \boldsymbol{I}-\frac{2}{3 J}\left(\frac{\partial W}{\partial \boldsymbol{C}^{*}}: \boldsymbol{C}^{*}\right) \boldsymbol{I}+\frac{2}{J} \boldsymbol{F}^{*} \frac{\partial W}{\partial \boldsymbol{C}^{*}} \boldsymbol{F}^{*^{T}},
\end{aligned}
$$

where : denotes the inner product and $\boldsymbol{F}^{*}=J^{-1 / 3} \boldsymbol{F}$. To ensure zero stress in the undeformed state it will be assumed that

$$
\frac{\partial W}{\partial J}(1, \boldsymbol{I})=0, \quad \frac{\partial W}{\partial \boldsymbol{C}^{*}}(1, \boldsymbol{I})=\frac{1}{3} \operatorname{tr}\left[\frac{\partial W}{\partial \boldsymbol{C}^{*}}(1, \boldsymbol{I})\right] \boldsymbol{I} .
$$


Given that $\operatorname{tr}\left(\boldsymbol{F}^{*} \frac{\partial W}{\partial \boldsymbol{C}^{*}} \boldsymbol{F}^{*^{T}}\right)=\frac{\partial W}{\partial \boldsymbol{C}^{*}}: \boldsymbol{C}^{*}$, it follows immediately that

$$
\operatorname{tr} \boldsymbol{\sigma}=3 \frac{\partial W}{\partial J}
$$

It is worthwhile emphasising that this results holds for all elastic materials and for all deformations. The Cauchy stress can now be additively decomposed into hydrostatic and deviatoric stress components as follows:

$$
\boldsymbol{\sigma}=\pi \boldsymbol{I}+\operatorname{dev} \boldsymbol{\sigma}
$$

where

$$
\begin{gathered}
\pi\left(J, \boldsymbol{C}^{*}\right)=\frac{\operatorname{tr} \boldsymbol{\sigma}}{3}=\frac{\partial W}{\partial J} \\
\operatorname{dev} \boldsymbol{\sigma}\left(J, \boldsymbol{F}^{*}\right)=\boldsymbol{\sigma}-\frac{1}{3} \operatorname{tr} \boldsymbol{\sigma} \boldsymbol{I}=\frac{2}{J}\left(\boldsymbol{F}^{*} \frac{\partial W}{\partial \boldsymbol{C}^{*}} \boldsymbol{F}^{*^{T}}-\frac{1}{3}\left(\frac{\partial W}{\partial \boldsymbol{C}^{*}}: \boldsymbol{C}^{*}\right) \boldsymbol{I}\right) .
\end{gathered}
$$

\section{The volumetric-isochoric split and hydrostatic ten- sion}

Consider now the volumetric-isochoric split

$$
W\left(J, \boldsymbol{C}^{*}\right)=f(J)+\mathcal{W}\left(\boldsymbol{C}^{*}\right)
$$

an assumption widely made when modelling slightly compressible materials, where the separate functionals are assumed infinitely differentiable and the initial conditions

$$
f(1)=0, \quad \mathcal{W}(\boldsymbol{I})=0,
$$

are imposed to ensure zero strain energy in the reference configuration. An immediate consequence of this decomposition is that the hydrostatic Cauchy stress is now only a 
function of the volume change and that the deviatoric Cauchy stress is decomposed into separate functions of the volume change and the isochoric deformation gradient tensor $\boldsymbol{F}^{*}$ as follows:

$$
\begin{aligned}
\pi\left(J, \boldsymbol{C}^{*}\right) & =f^{\prime}(J), \\
\operatorname{dev} \boldsymbol{\sigma}\left(J, \boldsymbol{F}^{*}\right) & =\frac{2}{J} \boldsymbol{\sigma}_{d}\left(\boldsymbol{F}^{*}\right), \boldsymbol{\sigma}_{d}\left(\boldsymbol{F}^{*}\right) \equiv \boldsymbol{F}^{*} \frac{d \mathcal{W}\left(\boldsymbol{C}^{*}\right)}{d \boldsymbol{C}^{*}} \boldsymbol{F}^{*^{T}}-\frac{1}{3} \frac{d \mathcal{W}\left(\boldsymbol{C}^{*}\right)}{d \boldsymbol{C}^{*}}: \boldsymbol{C}^{*} \boldsymbol{I},
\end{aligned}
$$

where the prime notation denotes differentiation with respect to the appropriate argument and, to ensure that the reference configuration is stress-free, it will be assumed that

$$
f^{\prime}(1)=0, \quad \operatorname{dev} \boldsymbol{\sigma}_{d}(\boldsymbol{I})=\mathbf{0} .
$$

This decoupling of the hydrostatic stress from the isochoric strain $\boldsymbol{C}^{*}$ and the factorisation of the deviatoric stress seem overly prescriptive for anisotropic materials.

Assume now a state of hydrostatic tension, with $\boldsymbol{\sigma}=\omega \boldsymbol{I}$, for which, by definition,

$$
\pi\left(J, \boldsymbol{C}^{*}\right)=\omega, \quad \operatorname{dev} \boldsymbol{\sigma}\left(J, \boldsymbol{F}^{*}\right)=\mathbf{0} .
$$

Substitution into (3.3) yields

$$
\omega=f^{\prime}(J), \quad \mathbf{0}=\frac{2}{J} \boldsymbol{\sigma}_{d}\left(\boldsymbol{F}^{*}\right) .
$$

The first of these determines the volume change in terms of the amount of hydrostatic tension. It follows from the initial condition $(3.4)_{2}$ that a solution of the second equation is given by $\boldsymbol{F}^{*}=\boldsymbol{I}$, which is a uniform dilatation. Therefore a material with a separable strain energy can behave as if it were an isotropic material under hydrostatic tension, irrespective of the assumed material symmetry. For infinitesimal strains, uniform dilatation is the unique solution to the problem of hydrostatic tension for all such elastic materials and it seems that the commercial Finite Element codes understandably step this uniform 
iff

$$
\operatorname{tr} \boldsymbol{\sigma}=F(J), \quad \text { arbitrary } F(.) .
$$

Proof. Assume that (1.1) holds. Then it follows from (2.5) that

$$
\operatorname{tr} \boldsymbol{\sigma}=3 f^{\prime}(J)
$$
spilt (1.1) behave isotropically under hydrostatic tension and compression.

Theorem. A strain-energy function has the additive decomposition

$$
W\left(J, \boldsymbol{C}^{*}\right)=f(J)+\mathcal{W}\left(\boldsymbol{C}^{*}\right),
$$

dilation behaviour for infinitesimal strains into the non-linear regime. This explains the deficiencies of the assumption (3.1) that have been highlighted elsewhere. The main argument advanced by Ní Annaidh et al. [4], Vergori et al. [6], Gilchrist et al. [7] and Nolan et al. [8], for example, is that Finite Element simulations of the mechanical response to hydrostatic tension using specific forms of anisotropy that assume (3.1) yield a purely isotropic strain response, with therefore no contribution from, for example, fibres, which are the components typically inducing anisotropy in non-linear materials. This seems unacceptable physically, since fibres, for example, are much stiffer than the matrix in which they are embedded. In light of the analysis presented here, this behaviour is not now unexpected. All slightly compressible elastic materials modelled using the additive

The mechanical response of bodies for which the hydrostatic stress depends only on the volume change can be summarised in the following equivalence theorem, a trivial consequence of the identity (2.5), which generalises previous results of, for example, Richter [12], Sansour [9] and Horgan and Murphy [10] for specific material symmetries:

Setting $F(J)=3 f^{\prime}(J)$ recovers $(3.6)$. 
Now assume that (3.6) holds. The identity (2.5) now yields

$$
3 \frac{\partial W}{\partial J}\left(J, \boldsymbol{C}^{*}\right)=F(J)
$$

A simple integration yields (1.1), with $f(J)=\int(1 / 3) F(J) d J$.

This equivalence result was first obtained by Charrier et al. [5] using a different method. It is worthwhile emphasising that this equivalence holds for all deformations of all (hyper)elastic materials. The seemingly intuitive appeal of (3.1), and this is surely another reason for its widespread adoption, is undermined by this equivalence relation: if one wanted to model the slight compressibility of elastic materials, one would surely not assume ab initio that $\operatorname{tr} \boldsymbol{\sigma}=F(J)$, especially when considering anisotropic materials.

Thus one is lead by consideration of hydrostatic tension to require that either $\frac{\partial \operatorname{tr} \boldsymbol{\sigma}}{\partial \boldsymbol{C}^{*}} \neq \mathbf{0}$, or, equivalently,

$$
\frac{\partial^{2} W}{\partial J \partial \boldsymbol{C}^{*}} \neq \mathbf{0}
$$

when modelling the slight compressibility of anisotropic materials.

Many of the results obtained here were previously obtained by Federico [2] but the approach and emphases here are different.

\section{Generalising the strain energy function}

There are two approaches that can be adopted to improve the standard model (1.1). One could generalise the assumption on the hydrostatic stress (3.6) to include a contribution from the isochoric strain tensor or, more immediately, one could generalise the form of the strain energy function. Because of the theorem of the last section, these two approaches are essentially equivalent and therefore only the second approach will be considered here. The method of Spencer [11] is adopted, one that explicitly utilises the fact that the volume changes are assumed infinitesimal for slightly compressible materials. Specifically, the strain energy function $W\left(J, \boldsymbol{C}^{*}\right)$ is expanded in a Taylor series about $J=1$ to the 
second power in $J-1$ as follows:

$$
W\left(J, \boldsymbol{C}^{*}\right)=W\left(1, \boldsymbol{C}^{*}\right)+(J-1) \frac{\partial W}{\partial J}\left(1, \boldsymbol{C}^{*}\right)+\frac{1}{2}(J-1)^{2} W_{J J}\left(1, \boldsymbol{C}^{*}\right) .
$$

Truncation after the second order term seems reasonable, assuming that the $W_{J J}$ term is of the order of the bulk modulus $\kappa$, with all other coefficients of the volumetric terms assumed to be of an order less than or equal to this term. Indeed the $W\left(1, C^{*}\right)$ and the $\frac{\partial W}{\partial J}\left(1, \boldsymbol{C}^{*}\right)$ terms here are assumed to be of the order of a typical shear modulus of the material, $\mu$, with the volume change assumed to be of $\mathcal{O}(\mu / \kappa)$. Thus, if the strain-energy function is non-dimensionalised with respect to the shear modulus, the first term in the Taylor series expansion can be considered as the zero-order term and the remaining terms the first-order terms in a perturbation series in the parameter $\mu / \kappa$.

Applying (1.2) to (4.1) yields $W\left(1, \boldsymbol{C}^{*}\right)=W_{i}\left(\boldsymbol{C}^{*}\right)$, noting that $W_{i}(\boldsymbol{C})$ is a known incompressible strain-energy function. For convenience, let $\mathcal{F}\left(\boldsymbol{C}^{*}\right) \equiv \frac{\partial W}{\partial J}\left(1, \boldsymbol{C}^{*}\right), \mathcal{G}\left(\boldsymbol{C}^{*}\right) \equiv$ $W_{J J}\left(1, C^{*}\right)$ and the proposed model therefore has the form

$$
W\left(J, \boldsymbol{C}^{*}\right)=W_{i}\left(\boldsymbol{C}^{*}\right)+(J-1) \mathcal{F}\left(\boldsymbol{C}^{*}\right)+\frac{1}{2}(J-1)^{2} \mathcal{G}\left(\boldsymbol{C}^{*}\right) .
$$

Some rationale for the choice of $\mathcal{F}, \mathcal{G}$ is needed if (4.2) is to be a workable model of slight compressibility. Some preliminary guidance is given by the restrictions that should be imposed on every candidate strain energy. First note that if $W_{i}(\boldsymbol{I})=0$, then the strain energy is zero in the reference configuration. Substituting (4.2) into the initial conditions (2.4) for zero stress in the reference configuration yields

$$
\mathcal{F}(\boldsymbol{I})=0, \quad W_{i}^{\prime}(\boldsymbol{I})=\frac{1}{3} \operatorname{tr}\left[W_{i}^{\prime}(\boldsymbol{I})\right] \boldsymbol{I} .
$$

The compressibility condition (3.7) requires that

$$
\mathcal{F}^{\prime}(\boldsymbol{I}) \neq 0
$$


Therefore $\mathcal{F}\left(\boldsymbol{C}^{*}\right)$ cannot be constant and, in particular, cannot be identically zero, as is assumed in the separable model of slight compressibility, (3.1). Motivated by a desire to keep mathematical models as simple as possible, it is therefore proposed that $\mathcal{F}\left(\boldsymbol{C}^{*}\right)$ be a linear function of the appropriate invariants of $\boldsymbol{C}^{*}$. Comparing the first two terms in (4.2) shows that the complexity of the model in $\boldsymbol{C}^{*}$ is reduced when we include the linear term in the volume change. Keeping this structure in mind, it will be additionally assumed that $\mathcal{G}\left(\boldsymbol{C}^{*}\right)$ is a positive constant $(=c)$ and therefore the proposed model of slight compressibilty has the form

$$
W\left(J, \boldsymbol{C}^{*}\right)=W_{i}\left(\boldsymbol{C}^{*}\right)+(J-1) \mathcal{F}\left(\boldsymbol{C}^{*}\right)+\frac{c}{2}(J-1)^{2},
$$

with, from (2.5),

$$
\operatorname{tr} \boldsymbol{\sigma}=\mathcal{F}\left(\boldsymbol{C}^{*}\right)+c(J-1) .
$$

Some additional simplicity in the linear form of $\mathcal{F}\left(\boldsymbol{C}^{*}\right)$ is assumed here. Recalling the centrality of the problem of hydrostatic tension in the argument for a new accounting for slight compressibility, it is proposed that $\mathcal{F}\left(\boldsymbol{C}^{*}\right)$ is linear in the smallest subset of invariants in $\boldsymbol{C}^{*}$ that ensure that the hydrostatic stress for slight compressibility is compatible with the hydrostatic stress for the linear theory, on restriction to infinitesimal strains. Examples of the application of this procedure will be given in the next section for isotropic, transversely isotropic and orthotropic materials. The reasons for this are twofold: (1) every well-posed non-linear theory should recover its linear form on restriction to infinitesimal inputs (Quintanilla and Saccomandi [3]) and (2) if a slightly compressible material is locally subjected to hydrostatic tension in simulations of practical problems, for which the applied stresses will be of the order of a typical shear modulus of the material, the linear theory becomes applicable as the volume change is assumed infinitesimal.

To determine the material constants in (4.3), it is proposed that experiments where the principal Cauchy stresses are known, such as uniaxial and biaxial tension, be per- 
To illustrate the ideas of the last section for the new model of slight compressibility proposed here, appropriate forms of slight compressibility for isotropic, transversely isotropic and orthotropic materials will now be considered. A key feature of the proposed model is that the linear theory in each case is recovered on restriction to infinitesimal deformations. To consider infinitesimal strains, assume that

$$
\boldsymbol{F}=\boldsymbol{I}+\boldsymbol{H}, \quad h \equiv \sqrt{\boldsymbol{H}: \boldsymbol{H}} \ll 1,
$$

where $\boldsymbol{H}$ is the displacement gradient tensor. Then, neglecting here and hereafter higher order terms,

$$
J=1+\operatorname{tr} \boldsymbol{\epsilon},
$$



order term in $\boldsymbol{H}$

$$
\begin{array}{ll}
\boldsymbol{F}^{*}=J^{-1 / 3} \boldsymbol{F}=\boldsymbol{I}+\boldsymbol{H}^{*}, & \boldsymbol{H}^{*} \equiv \boldsymbol{H}-\frac{1}{3} \operatorname{tr} \boldsymbol{\epsilon}, \\
\boldsymbol{C}^{*}=J^{-2 / 3} \boldsymbol{C}=\boldsymbol{I}+2 \boldsymbol{\epsilon}^{*}, & \boldsymbol{\epsilon}^{*} \equiv \boldsymbol{\epsilon}-\frac{1}{3} \operatorname{tr} \boldsymbol{\epsilon} .
\end{array}
$$

Since $\mathcal{F}\left(\boldsymbol{C}^{*}\right)$ is assumed linear in the invariants of $\boldsymbol{C}^{*}$ the hydrostatic stress becomes

$$
\operatorname{tr} \boldsymbol{\sigma}=c_{1}\left(I_{1}^{*}-3\right)+c_{2}\left(I_{2}^{*}-3\right)+c(J-1) .
$$

On restriction to infinitesimal deformations and truncating after first order terms in $h$,

$$
\operatorname{tr} \boldsymbol{\sigma}=c \operatorname{tr} \boldsymbol{\epsilon} .
$$

The linear theory for isotropic material yields

$$
\operatorname{tr} \boldsymbol{\sigma}=(3 \lambda+2 \mu) \operatorname{tr} \boldsymbol{\epsilon}
$$

where $\lambda, \mu$ are the Lamé constants. A comparison of these two linear forms for the hydrostatic stress shows that the simplest form of the non-linear hydrostatic stress (5.3) that is compatible with the linear is the choice

$$
c_{1}=c_{2}=0, \quad c=3 \lambda+2 \mu,
$$


On restriction to infinitesimal deformations, the hydrostatic stress is therefore

$$
\operatorname{tr} \boldsymbol{\sigma}=\left(c-\frac{2}{3} c_{1}-\frac{4}{3} c_{2}\right) \operatorname{tr} \boldsymbol{\epsilon}+2\left(c_{1}+2 c_{2}\right) \boldsymbol{M} . \boldsymbol{\epsilon} \boldsymbol{M}
$$

The linear theory for transversely isotropic materials (see, for example, Spencer [16]) that

$$
\operatorname{tr} \boldsymbol{\sigma}=\left(3 \lambda+2 \mu_{T}+\alpha\right) \operatorname{tr} \boldsymbol{\epsilon}+\left(3 \alpha+\beta+4\left[\mu_{L}-\mu_{T}\right]\right) \boldsymbol{M .} \boldsymbol{\epsilon} \boldsymbol{M},
$$

which has the separable form (1.1) and therefore the volumetric-isochoric split seems acceptable for isotropic materials.

\subsection{Transversely isotropic materials}

For these materials the general strain energy has the from $W=W\left(J, I_{1}^{*}, I_{2}^{*}, I_{4}^{*}, I_{5}^{*}\right)$, where $I_{1}^{*}, I_{2}^{*}$ are given in $(5.2)$ and

$$
I_{4}^{*}=M \cdot C^{*} M, \quad I_{5}^{*}=M \cdot\left(C^{*}\right)^{2} M
$$

where $\boldsymbol{M}$ is the preferred direction of the materials. The initial assumption for $\mathcal{F}\left(\boldsymbol{C}^{*}\right)$ is therefore that

$$
\mathcal{F}\left(\boldsymbol{C}^{*}\right)=c_{1}\left(I_{4}^{*}-1\right)+c_{2}\left(I_{5}^{*}-1\right),
$$

noting that the there are no terms linear in the isotropic invariants following the analysis of the last subsection and therefore now

$$
\operatorname{tr} \boldsymbol{\sigma}=c_{1}\left(I_{4}^{*}-1\right)+c_{2}\left(I_{5}^{*}-1\right)+c(J-1) .
$$




$$
\begin{aligned}
& W\left(J, \boldsymbol{C}^{*}\right)=W_{i}\left(\boldsymbol{C}^{*}\right)+c_{1}(J-1)\left(I_{4}^{*}-1\right)+\frac{c}{2}(J-1)^{2}, \\
& W\left(J, \boldsymbol{C}^{*}\right)=W_{i}\left(\boldsymbol{C}^{*}\right)+c_{1}(J-1)\left(I_{5}^{*}-1\right)+\frac{c}{2}(J-1)^{2},
\end{aligned}
$$

where the constants $c_{1}, c$ are to determined from volume change measurements during characterisation tests.

\subsection{Materials with two families of mechanically equivalent fi- bres}

Denote the two preferred directions by $\boldsymbol{M}, \boldsymbol{M}^{\prime}$, with now $W=W\left(J, I_{1}^{*}, I_{2}^{*}, I_{4}^{*}, I_{5}^{*}, I_{6}^{*}, I_{7}^{*}, I_{8}^{*}\right)$, where $I_{1}^{*}, I_{2}^{*}, I_{4}^{*}, I_{5}^{*}$ have been defined previously and

$$
I_{6}^{*}=M^{\prime} \cdot C^{*} M^{\prime}, \quad I_{7}^{*}=M^{\prime} \cdot\left(C^{*}\right)^{2} M^{\prime}, \quad I_{8}^{*}=M \cdot M^{\prime} M \cdot C^{*} M^{\prime} .
$$

Bearing in mind that the fibres are mechanically equivalent, assume initially therefore that

$$
\mathcal{F}\left(\boldsymbol{C}^{*}\right)=c_{1}\left(I_{4}^{*}+I_{6}^{*}-2\right)+c_{2}\left(I_{5}^{*}+I_{7}^{*}-2\right)+c_{3}\left(I_{8}^{*}-\left(\boldsymbol{M} \cdot \boldsymbol{M}^{\prime}\right)^{2}\right),
$$

with now

$$
\operatorname{tr} \boldsymbol{\sigma}=c_{1}\left(I_{4}^{*}+I_{6}^{*}-2\right)+c_{2}\left(I_{5}^{*}+I_{7}^{*}-2\right)+c_{3}\left(I_{8}^{*}-\left(\boldsymbol{M} \cdot \boldsymbol{M}^{\prime}\right)^{2}\right)+c(J-1) .
$$


On restriction to infinitesimal deformations therefore

$$
\begin{gathered}
\operatorname{tr} \boldsymbol{\sigma}=2\left(c_{1}+2 c_{2}\right)\left(\boldsymbol{M} . \boldsymbol{\epsilon} \boldsymbol{M}+\boldsymbol{M}^{\prime} . \boldsymbol{\epsilon} \boldsymbol{M}^{\prime}-\frac{2}{3} \operatorname{tr} \boldsymbol{\epsilon}\right)+2 c_{3} \boldsymbol{M} . \boldsymbol{M}^{\prime}\left(\boldsymbol{M} . \boldsymbol{\epsilon} \boldsymbol{M}^{\prime}-\frac{1}{3} \boldsymbol{M} . \boldsymbol{M}^{\prime} \operatorname{tr} \boldsymbol{\epsilon}\right)+c \operatorname{tr} \boldsymbol{\epsilon} \\
=\operatorname{tr} \boldsymbol{\epsilon}\left(c-\frac{4}{3}\left(c_{1}+2 c_{2}\right)-\frac{2}{3} c_{5}\left(\boldsymbol{M} . \boldsymbol{M}^{\prime}\right)^{2}\right)+2\left(c_{1}+2 c_{2}\right)\left(\boldsymbol{M} . \boldsymbol{\epsilon} \boldsymbol{M}+\boldsymbol{M}^{\prime} \cdot \boldsymbol{\epsilon} \boldsymbol{M}^{\prime}\right) \\
+2 c_{3} \boldsymbol{M} . \boldsymbol{M}^{\prime} \boldsymbol{M} . \boldsymbol{\epsilon} \boldsymbol{M}^{\prime}
\end{gathered}
$$

In the corresponding linear theory (see, for example, Equation (45) of Spencer [16] and using his notation)

$$
\begin{aligned}
& \operatorname{tr} \boldsymbol{\sigma}=\left(3 \lambda+2 \mu_{T}+2 \gamma_{3}+\gamma_{4}\left(\boldsymbol{M} . \boldsymbol{M}^{\prime}\right)^{2}\right) \operatorname{tr} \boldsymbol{\epsilon} \\
& \left(\boldsymbol{M} . \boldsymbol{\epsilon} \boldsymbol{M}+\boldsymbol{M}^{\prime} \cdot \boldsymbol{\epsilon} \boldsymbol{M}^{\prime}\right)\left(2 \gamma_{1}+3 \gamma_{3}+\gamma_{5}\left(\boldsymbol{M} \cdot \boldsymbol{M}^{\prime}\right)^{2}+\gamma_{6}+2 \gamma_{7}\right)+ \\
& \left(\gamma_{2} \boldsymbol{M} . \boldsymbol{M}^{\prime}+2 \gamma_{5}+3 \gamma_{6}\right) \boldsymbol{M} . \boldsymbol{M}^{\prime} \boldsymbol{M} . \boldsymbol{\epsilon} \boldsymbol{M}^{\prime} .
\end{aligned}
$$

Comparing (5.11) 2 and (5.12) shows that to fully recover the linear theory, it is required that

$$
c_{3} \neq 0 \text {, }
$$

and that one of $c_{1}, c_{2}$ can be set equal to zero, in order to simplify the complexity of the model. Thus, adopting an obvious change of notation for the material constants, either of

$$
\begin{aligned}
& W\left(J, \boldsymbol{C}^{*}\right)=W_{i}\left(\boldsymbol{C}^{*}\right)+c_{1}(J-1)\left(I_{4}^{*}+I_{6}^{*}-2\right)+c_{3}(J-1)\left(I_{8}^{*}-\left(\boldsymbol{M} \cdot \boldsymbol{M}^{\prime}\right)^{2}\right)+\frac{c}{2}(J-1)^{2}, \\
& W\left(J, \boldsymbol{C}^{*}\right)=W_{i}\left(\boldsymbol{C}^{*}\right)+c_{1}(J-1)\left(I_{5}^{*}+I_{7}^{*}-2\right)+c_{3}(J-1)\left(I_{8}^{*}-\left(\boldsymbol{M} \cdot \boldsymbol{M}^{\prime}\right)^{2}\right)+\frac{c}{2}(J-1)^{2},
\end{aligned}
$$

seems a reasonable model of slight compressibility for orthotropic materials, adopting the motivation proposed earlier. Note the necessity of including an $I_{8}^{*}$ term in the proposed model for slight compressibility, a term which is absent in the model of Nolan et al. [8], for example. 


\section{Acknowledgements}

The authors would like to thank Professor P. Neff for his insight and encouragement. The authors are also grateful to the anonymous referees whose comments have resulted in a significantly improved final version of this paper.

\section{References}

[1] Flory PJ (1961) Thermodynamic relations for high elastic materials. Transactions of the Faraday Society, 57, 829-838.

[2] Federico S (2010) Volumetric-Distortional Decomposition of Deformation and Elasticity Tensor. Mathematics and Mechanics of Solids, 15, 672-690.

[3] Quintanilla R, Saccomandi G (2007) The importance of the compatibility of nonlinear constitutive theories with their linear counterparts. J. Appl. Mech., 74, 455-460.

[4] Ní Annaidh A, Destrade M, Gilchrist MD, Murphy JG (2013) Deficiencies in numerical models of anisotropic nonlinearly elastic materials, Biomechanics and Modeling in Mechanobiology, 12, 781-791.

[5] Charrier P, Dacorogna B, Hanouzet B, Laborde P (1988). An existence theorem for slightly compressible materials in nonlinear elasticity. SIAM Journal on Mathematical Analysis, 19, 70-85.

[6] Vergori L, Destrade M, McGarry P, Ogden RW (2013) On anisotropic elasticity and questions concerning its Finite Element implementation, Comput. Mech., 52, $1185-1197$.

[7] Gilchrist MD, Murphy JG, Parnell W, Pierrat B (2014) Modelling the slight compressibility of anisotropic soft tissue, Int. J. Solids Struct., 51, 3857-3865. 
[8] Nolan DR, Gower AL, Destrade M, Ogden RW, McGarry JP (2014) A robust anisotropic hyperelastic formulation for the modelling of soft tissue, Journal of the Mechanical Behavior of Biomedical Materials, 39, 48-60.

[9] Sansour C (2008) On the physical assumptions underlying the volumetric-isochoric split and the case of anisotropy. Eur J Mech A Solids, 27, 28-39.

[10] Horgan CO, Murphy JG (2009) Constitutive modeling for moderate deformations of slightly compressible rubber. J. Rheol., 53,153-168.

[11] Spencer AJM (1964) Finite deformations of an almost incompressible elastic solid. in Second-Order Effects in Elasticity, Plasticity and Fluid Dynamics (Eds. M. Reiner and D. Abir). Macmillan, New York.

[12] Richter H (1948) Das isotrope Elastizitätsgesetz. ZAMM-Journal of Applied Mathematics and Mechanics/Zeitschrift fr Angewandte Mathematik und Mechanik, 28, 205-209.

[13] Hartmann S, Neff P (2003) Polyconvexity of generalized polynomial-type hyperelastic strain energy functions for near-incompressibility. International Journal of Solids and Structures, 40, 2767-2791.

[14] Abaqus 6.13 Documentation (2013) Dassault Systèmes Simulia Corp., Providence, RI, USA.

[15] Holzapfel GA (2006) Determination of material models for arterial walls from uniaxial extension tests and histological structure. Journal of Theoretical Biology, 238, 290-302.

[16] Spencer AJM (1984) Constitutive theory for strongly anisotropic solids. In Continuum Theory of the Mechanics of Fibre-reinforced Composites (Ed. A.J.M. Spencer). CISM Courses and Lectures No. 282. Vienna: Springer Verlag. 